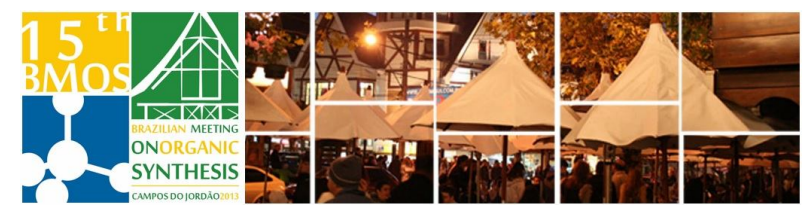

\title{
Diastereoselective synthesis of dihydroquinolines and tetrahydroquinolines from Morita Baylis-Hillman adducts
}

\section{Manoel T. Rodrigues Jr., Andre Capretz Agy, Danilo Machado Lustosa and Fernando Coelho*}

\author{
Laboratório de Síntese de Produtos Naturais e Fármacos, Instituto de Química, Universidade Estadual de \\ Campinas-UNICAMP, Caixa Postal 6154, 13083-970, Campinas-SP-Brazil \\ *coelho@iqm.unicamp.br
}

Keywords: Morita-Baylis-Hillman Reaction, dihydroquinolines and tetrahydroquinolines.

\section{INTRODUCTION}

The quinolone, dihydroquinolines and tetrahydroquinolines unities are present in many natural and synthetic compounds. These nuclei exhibit a wide spectrum of medicinal properties, such as antimalarial, antitumor, antibacterial, antithrombin, and many others. ${ }^{1}$ Due to this biological profile several synthetic approaches have been known for the preparation of these heterocycles. ${ }^{2}$ In this work we disclosed a facile method to prepare quinolines derivatives through a Morita-BaylisHillman adduct. Our approach is based on a tandem sequence involving a Michael addition reaction and a $S_{N} A r$ reaction.

\section{RESULTS AND DISCUSSION}

2-Bromo-3-nitrobenzaldehyde (3) was prepared according to a procedure described in literature. Acid 1 was reduced in the presence of $\mathrm{BH}_{3} . \mathrm{SMe}_{2}$ to afford alcohol 2 (Scheme 1). Oxidation of the alcohol 2 to aldehyde was accomplished using PCC, in 91\% overall yield (2 steps). MBH adduct 4 was prepared using a protocol developed by our group. ${ }^{3}$ In brief, aldehydes were treated with methyl acrylate to provide the corresponding $\mathrm{MBH}$ adduct in $98 \%$ yield. Compound 4 was therefore silylated in the presence of TBSOTf to give silylated derivative 5 , in $95 \%$ yield (Scheme 1).

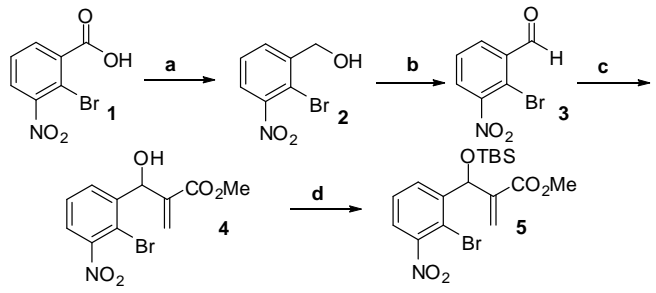

Scheme 1. Preparation of aldehyde 3 and MBH adduct 4 and silylation of the $\mathrm{MBH}$ adduct. a) $\mathrm{BH}_{3}$. $\mathrm{SMe}_{2}$, rt, 12h; b) PCC, $\mathrm{CH}_{2} \mathrm{Cl}_{2}$, rt, 12h (91\% - 2 steps); c) Methyl acrilate, DABCO, ))), 24h, 98\%; d) TBSOTf, $\mathrm{CH}_{2} \mathrm{Cl}_{2}$, rt, $2 \mathrm{~h}, 95 \%$.

A solution of the silylated MBH adduct 5 in methanol was treated, under reflux, with amine (1.2 eq.) in the presence of triethylamine (acting as a base) to give the substituted quinolines derivatives in good syn diastereoselectivity (syn/anti) and yields (Table 1).

Table 1. Synthesis of dihydro- and tetrahydroquinolines

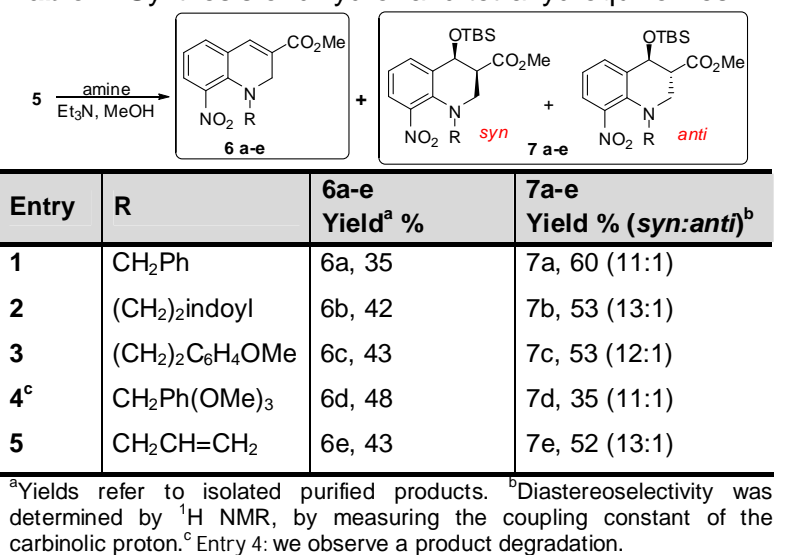

The formation of compounds 6a-e can be explained by a $\mathrm{S}_{\mathrm{N}} 2^{\prime}$ displacement reaction of silylated $\mathrm{MBH} \mathbf{5}$ with amine followed by cyclization. Otherwise, the high diastereoselectivity attained in the preparation of compounds 7a-e can be explained by the control exerted by the voluminous silyl group. Previous results described by our group support this proposition. Unfortunately all chromatographic attempts to separate the diastereoisomers failed. ${ }^{4}$

\section{CONCLUSION}

In summary, we have demonstrated that dihydroand tetrahydroquinolines can be easily prepared in good yields and diastereoselectivity from $\mathrm{MBH}$ adducts.

\section{ACKNOWLEDGEMENTS}

We thank FAPESP, CNPq and CAPES for financial support.

\section{REFERENCES}

1 Kaur, K.; Jain, M.; Reddy, R. P.; Jain, R. Eur. J. Med. Chem. 2010, 45 3245. 2Madapa, S.; Tusi, Z.; Batra, S. Curr. Org. Chem. 2008, 12,1116.

${ }^{3}$ Coelho, F.; Almeida, W. P.; Mateus, C. R.; Feltrin, M.; Costa, A. M. Tetrahedron 2001, 57, 6901. . . Rodrigues Jr., M. T.; Gomes, J. C.; Smith, J.; Coelho, F. Tetrahedron Lett. 2010, 51, 4988. 